\title{
A Certain Subclass of Analytic Functions
}

\author{
Young Jae Sim, Oh Sang Kwon* \\ Department of Mathematics, Kyungsung University, Busan, Korea (South) \\ Email: \{yjsim, *oskwon\}@ks.ac.kr
}

Received February 20, 2012; revised April 20, 2012; accepted April 28, 2012

\begin{abstract}
In the present paper, we introduce a class of analytic functions in the open unit disc by using the analytic function $q_{\alpha}(z)=3 /\left(3+(\alpha-3) z-\alpha z^{2}\right)$, which was investigated by Sokó [1]. We find some properties including the growth theorem or the coefficient problem of this class and we find some relation with this new class and the class of convex functions.
\end{abstract}

Keywords: Univalent Functions; Convex Functions; Subordination; Order of Convexity

\section{Introduction}

Let $H$ denote the class of analytic functions in the unit disc $\mathbb{U}=\{z:|z|<1\}$ on the complex plane $\mathbb{C}$. Let $A$ denote the subclass of $H$ consisting of functions normalized by $f(0)=0$ and $f^{\prime}(0)=1$. The set of all functions $f \in A$ that are convex univalent in $\mathbb{U}$ by $K$. Recall that a set $E$ is said to be convex if and only if the linear segment joining any two points of $E$ lies entirely in $E$. Let the function $f$ be analytic univalent in the unit disc $\mathbb{U}$ on the complex plane $\mathbb{C}$ with the normalization. Then $f$ maps $\mathbb{U}$ onto a convex domain $E$ if and only if

$$
\operatorname{Re}\left\{1+\frac{z f^{\prime \prime}(z)}{f^{\prime}(z)}\right\}>0(z \in U) \text {. }
$$

Robertson introduced in [2], the class $K(\alpha)$ of convex functions of order $\alpha(\alpha \leq 1)$, which is defined by

$$
K(\alpha)=\left\{f \in A: \operatorname{Re}\left(1+\frac{z f^{\prime \prime}(z)}{f^{\prime}(z)}\right)>\alpha, z \in \mathbb{U}\right\} .
$$

If $\alpha \in[0,1)$, then a function of this set is univalent and if $\alpha<0$ it may fail to be univalent. We denote $K(0)=K$. Let $S$ be denote the subset of $A$ which is composed of univalent functions. We say that $f$ is subordinate to $F$ in $\mathbb{U}$, written as $f \prec F$, if and only if, $f(z)=F(w(z))$ for some Schwarz function $w(z)$, $w(0)=0$ and $|w(z)|<1, z \in \mathbb{U}$. The class of convex functions $K$ can be defined in several ways, for example we say that $f$ is convex if it satisfies the condition

$$
1+\frac{z f^{\prime \prime}(z)}{f^{\prime}(z)} \prec \frac{1+z}{1-z} .
$$

${ }^{*}$ Corresponding author.
Many subclass of $K$ have been defined by the condition (1) with a convex univalent function $p$, given arbitrary, instead of the functions $(1+z) /(1-z)$. Janowski considered the function $p$, which maps the unit disc onto a disc in $[3,4]$. An interesting case when the function $p$ is convex but is not univalent was considered in [5]. A function $p$ that is not univalent and is not convex and maps unit circle onto a concave set was considered in [1]

Now, we shall introduce the class of analytic functions used in the sequel.

Definition 1.1. The function $f \in A$ belongs to the class $S Q(\alpha), \alpha \in(-3,1]$, if it satisfies the condition

$$
\sqrt{f^{\prime}(z)} \prec q_{\alpha}(z)=\frac{3}{3+(\alpha-3) z-\alpha z^{2}}
$$

Let the function $q_{\alpha}$ be given by (2). We note that

$$
\begin{aligned}
q_{\alpha}(z) & =3 /(3+(\alpha-3) z-\alpha z)=\frac{3}{3+\alpha}\left[\frac{1}{1-z}+\frac{\alpha}{\alpha z+3}\right] \\
& =1+\frac{(3-\alpha)^{2}}{3(3+\alpha)} z+\cdots
\end{aligned}
$$

Sokó investigated in [1] that the image of the unit circle $|z|=1$ under the function $q_{\alpha}$ is a curve described by

$$
\Gamma:(x-a)\left(x^{2}+y^{2}\right)-k\left(x-\frac{1}{2}\right)^{2}=0,
$$

where

$$
x=\operatorname{Re}\left\{q_{\alpha}\left(e^{i \phi}\right)\right\} \text { and } y=\operatorname{Im}\left\{q_{\alpha}\left(e^{i \phi}\right)\right\} \text {, }
$$

with $\phi \in(0,2 \pi)$ and $a=\frac{9(1+\alpha)}{2(3+\alpha)^{2}}$ and 


$$
k=\frac{54}{(3+\alpha)^{2}(3-\alpha)} \text {. }
$$

Thus the curve $\Gamma$ is symmetric with respect to real axis and $q_{\alpha}\left(e^{i \phi}\right)$ satisfies

$$
\frac{9(1+\alpha)}{2(3+\alpha)^{2}}<\operatorname{Re}\left(q_{\alpha}\left(e^{i \phi}\right)\right) \leq \frac{3}{2(3-\alpha)},
$$

where $\phi \in(0,2 \pi)$.

Especially, if $\alpha=0$, then $q_{0}(z)=1 /(1-z)$, which maps $\mathbb{U}$ onto the right of line $x=1 / 2$. And we note that if $-3<\alpha_{2}<\alpha_{1} \leq-1$, then $q_{\alpha_{1}} \prec q_{\alpha_{2}}$.

\section{Some Properties of Functions in $S Q(\alpha)$}

Now we shall find some properties of functions in the class $S Q(\alpha)$.

Theorem 2.1. If a function $f$ belongs to the class $S Q(\alpha), \alpha \in(-3,1]$, then there exists a function $g \in A$ such that

$$
\sqrt{g^{\prime}(z)} \prec 1 /(1-z)
$$

and a function $h \in A$ such that

$$
\sqrt{h^{\prime}(z)} \prec 3 /(3+\alpha z)
$$

and

$$
f^{\prime}(z)=g^{\prime}(z) h^{\prime}(z) .
$$

Proof. Let $f$ be in $S Q(\alpha)$. Then there exists an analytic function $w(z)$ with $w(0)=0$ and $|w(z)|<1$ for $z \in \mathbb{U}$ such that

$$
\sqrt{f^{\prime}(z)}=\frac{3}{(1-w(z))(3+\alpha w(z))} .
$$

From (4) we have

$$
\frac{f^{\prime \prime}(z)}{2 f^{\prime}(z)}=\frac{w^{\prime}(z)}{1-w(z)}-\frac{\alpha w^{\prime}(z)}{3+\alpha w(z)} .
$$

Define $g$ and $h$ so that

$$
\frac{g^{\prime \prime}(z)}{2 g^{\prime}(z)}=\frac{w^{\prime}(z)}{1-w(z)}
$$

and

$$
\frac{h^{\prime \prime}(z)}{2 h^{\prime}(z)}=-\frac{\alpha w^{\prime}(z)}{3+\alpha w^{\prime}(z)},
$$

respectively. Then

$$
\begin{gathered}
\sqrt{g^{\prime}(z)} \prec 1 /(1-z), \\
\sqrt{h^{\prime}(z)} \prec 3 /(3+\alpha z)
\end{gathered}
$$

and

$$
\frac{f^{\prime \prime}(z)}{2 f^{\prime}(z)}=\frac{g^{\prime \prime}(z)}{2 g^{\prime}(z)}+\frac{h^{\prime \prime}(z)}{2 h^{\prime}(z)} .
$$

Hence $f^{\prime}(z)=g^{\prime}(z) h^{\prime}(z)$, which proves Theorem 2.1 .

Theorem 2.2. If $f \in S Q(\alpha), \alpha \in(-3,1]$ and $|z|=r$, $0 \leq r<1$, then

$$
\begin{aligned}
\frac{1}{(1+r)^{2}(1+(|\alpha| / 3) r)^{2}} & \leq\left|f^{\prime}(z)\right| \\
& \leq \frac{1}{(1-r)^{2}(1-(|\alpha| / 3) r)^{2}}
\end{aligned}
$$

Proof. Suppose that $f \in S Q(\alpha)$. Then

$$
f^{\prime}(z)=g^{\prime}(z) h^{\prime}(z)
$$

For some $g$ and $h$ such that

$$
\sqrt{g^{\prime}(z)} \prec 1 /(1-z)
$$

and

$$
\sqrt{h^{\prime}(z)} \prec 3 /(3+\alpha z),
$$

respectively. And above subordination equations imply that

$$
\frac{1}{(1+r)^{2}} \leq\left|g^{\prime}(z)\right| \leq \frac{1}{(1-r)^{2}}
$$

and

$$
\frac{1}{(1+(|\alpha| / 3) r)^{2}} \leq\left|h^{\prime}(z)\right| \leq \frac{1}{(1-(|\alpha| / 3) r)^{2}},
$$

respectively. Since $f^{\prime}(z)=g^{\prime}(z) h^{\prime}(z)$, the modulus of $f^{\prime}(z)$ satisfies the inequality (5).

Next, we shall solve some coefficient problem for a special function to be in the class $S Q(\alpha)$.

Theorem 2.3. The function $g_{n}(z)=z+c z^{n}$ belongs to the class $S Q(\alpha)$, whenever

$$
|c|<\frac{27-24 \alpha+4 \alpha^{2}}{4 n(3-\alpha)^{2}} .
$$

Proof. Since $g_{n}^{\prime}(z)=1+n c z^{n-1}$, if we put

$$
G(z)=\sqrt{g_{n}^{\prime}(z)},
$$

then

$$
G^{2}(z)-1=n c z^{n-1} .
$$

Hence for $z \in \mathbb{U}$,

$$
\left|G^{2}(z)-1\right|<n|C|
$$

Since $\operatorname{Re}(G(z))>\sqrt{1-n|c|}$, if 


$$
\frac{3}{2(3-\alpha)}<\sqrt{1-n|c|},
$$

Then $g_{n}(z) \in S Q(\alpha)$ and we can easily derive that the inequality (6) is equivalent to

$$
|c|<\frac{27-24 \alpha+4 \alpha^{2}}{4 n(3-\alpha)^{2}} \text {. }
$$

\section{The Relations of the Classes $S Q$ and $K$}

It is well-known that the following implication holds:

$$
\operatorname{Re}\left\{1+\frac{z f^{\prime \prime}(z)}{f^{\prime}(z)}\right\}>0 \Rightarrow \operatorname{Re} \sqrt{f^{\prime}(z)}>\frac{1}{2} .
$$

More generally, the above implication (7) is can be generalized as following:

$$
\frac{z f^{\prime \prime}(z)}{f^{\prime}(z)} \prec \frac{z k^{\prime \prime}(z)}{k^{\prime}(z)} \Rightarrow f^{\prime}(z) \prec k^{\prime}(z) .
$$

Evidently, the implication (7) implies the relation $K(0) \subset S Q(1 / 2)$. In this chapter, we find some general relation between the classes $K(\alpha)$ and $S Q(\alpha)$.

Let us denote by $Q$ the class of functions $f$ that are analytic and injective on $\overline{\mathbb{U}}-E(f)$, where

$$
E(f)=\left\{\varsigma: \varsigma \in \partial \mathbb{U} \text { and } \lim _{z \rightarrow \varsigma} f(z)=\infty\right\}
$$

and are such that

$$
f^{\prime}(\varsigma) \neq 0(\varsigma \in \partial \mathbb{U}-E(f)) .
$$

Lemma 3.1. [6] Let $p \in Q$ with $p(0)=a$ and let

$$
q(z)=a+a_{n} z^{n}+\cdots
$$

Be analytic in $\mathbb{U}$ with

$$
q(z) \not z a \text { and } n \in \mathbb{N} .
$$

If $q$ is not subordinate to $p$, then there exist points

$$
z_{0}=r_{0} e^{i \theta} \in \mathbb{U} \text { and } \varsigma \in \partial \mathbb{U}-E(f),
$$

and there exists a number $m \geq n$ for which

$$
q\left(|z|<r_{0}\right) \subset p(\mathbb{U}), \quad q\left(z_{0}\right)=p(\varsigma)
$$

and

$$
z_{0} q^{\prime}\left(z_{0}\right)=m \varsigma p^{\prime}(\varsigma) .
$$

Theorem 3.2. Let $-1<\alpha<1$. If a function $f$ belongs to the class $A$ and

$$
f \operatorname{Re}\left\{1+\frac{z f^{\prime \prime}(z)}{f^{\prime}(z)}\right\}>\frac{2|\alpha|}{3-|\alpha|} \text { for } z \in \mathbb{U},
$$

then $f \in S Q(\alpha)$.

Proof. Suppose that $\alpha \neq 0$ and $f \notin S Q(\alpha)$ or equiva- lently,

$$
\sqrt{f^{\prime}(z)} \nprec q_{\alpha}(z) .
$$

Then by Lemma 3.1, there exist $z_{0} \in \mathbb{U}$ and $\varsigma \in \partial \mathbb{U}$, $\varsigma \neq 1$ and $m>1$ such that

$$
\sqrt{f^{\prime}\left(z_{0}\right)}=q_{\alpha}(\varsigma)
$$

and

$$
\left.z\left(\sqrt{f^{\prime}(z)}\right)^{\prime}\right|_{z=z_{0}}=m \varsigma q_{\alpha}^{\prime}(\varsigma) .
$$

Since

$$
\operatorname{Re}\left\{\frac{2 \varsigma-(\alpha+3) / \alpha}{\varsigma-1}\right\}=1+\frac{3+\alpha}{2 \alpha},
$$

For $|\varsigma|=1(\varsigma \neq 1)$,

$$
\begin{aligned}
& \operatorname{Re}\left\{1+\frac{z_{0} f^{\prime \prime}\left(z_{0}\right)}{f^{\prime}\left(z_{0}\right)}\right\} \\
= & \operatorname{Re}\left\{1+\frac{2 m \varsigma q_{\alpha}^{\prime}(\varsigma)}{q_{\alpha}(\varsigma)}\right\} \\
= & 1+2 m \operatorname{Re}\left\{-\frac{3(\alpha+3) / \alpha}{(\varsigma-1)(\alpha \varsigma+3)}-\frac{2 \varsigma-3(\alpha+3) / \alpha}{\varsigma-1}\right\} \\
= & 1+\frac{2 m(\alpha+3)}{\alpha} \operatorname{Re}\left\{\frac{-3}{(\varsigma-1)(\alpha \varsigma+3)}\right\} \\
& -2 m \operatorname{Re}\left\{\frac{2 \varsigma-3(\alpha+3) / \alpha}{\varsigma-1}\right\} \\
= & 1+\frac{2 m(\alpha+3)}{\alpha} \operatorname{Re}\left\{\frac{-3}{(\varsigma-1)(\alpha \varsigma+3)}\right\} \\
& -2 m \operatorname{Re}\left\{1+\frac{3+\alpha}{2 \alpha}\right\} .
\end{aligned}
$$

In case $-1<\alpha<0$, since the inequality (3) induces the following inequality:

$$
\begin{aligned}
& \frac{9(1+\alpha)}{2(3+\alpha)^{2}}<\operatorname{Re}\left\{\frac{-3}{(\varsigma-1)(\alpha \varsigma+3)}\right\} \leq \frac{3}{2(3-\alpha)}, \\
& \operatorname{Re}\left\{1+\frac{z_{0} f^{\prime \prime}\left(z_{0}\right)}{f^{\prime}\left(z_{0}\right)}\right\} \\
& \leq 1+\frac{2 m(\alpha+3)}{\alpha} \frac{9(1+\alpha)}{2(3+\alpha)^{2}}-2 m\left(1+\frac{3+\alpha}{2 \alpha}\right) \\
& =1+2 m\left(\frac{9(1+\alpha)}{2 \alpha(3+\alpha)}-1-\frac{3+\alpha}{2 \alpha}\right) \\
& \leq 1-\frac{3(1+\alpha)}{3+\alpha}=\frac{2|\alpha|}{3-|\alpha|},
\end{aligned}
$$


which is a contradiction to the hypothesis. In case $0<\alpha<1$, using the inequality (8) again,

$$
\begin{aligned}
& \operatorname{Re}\left\{1+\frac{z_{0} f^{\prime \prime}\left(z_{0}\right)}{f^{\prime}\left(z_{0}\right)}\right\} \\
& \leq 1+\frac{2 m(\alpha+3)}{\alpha} \frac{3}{2(3-\alpha)}-2 m\left(1+\frac{3+\alpha}{2 \alpha}\right) \\
& =1+2 m\left(\frac{3(3+\alpha)}{2 \alpha(3-\alpha)}-1-\frac{3+\alpha}{2 \alpha}\right) \\
& =1+\frac{3 m(\alpha-1)}{3-\alpha} \leq 1+\frac{3(\alpha-1)}{3-\alpha}=\frac{2|\alpha|}{3-|\alpha|},
\end{aligned}
$$

which is a contradiction to the hypothesis, hence

$$
\sqrt{f^{\prime}(z)} \prec q_{\alpha}(z) \text {, and } f \in S Q(\alpha) \text {. }
$$

If we put $\alpha=1 / 2$ in Theorem 3.2, we can get next Corollary.

Corollary 3.1. For $f \in A$, the following implication holds:

$$
\begin{aligned}
\operatorname{Re}\left\{1+\frac{z f^{\prime \prime}(z)}{f^{\prime}(z)}\right\} & >\frac{2}{5} \Rightarrow f \in S Q(1 / 2) \\
& \Rightarrow \operatorname{Re}\left\{\sqrt{f^{\prime}(z)}\right\}>\frac{27}{49} .
\end{aligned}
$$

Theorem 3.3. Let $\alpha \in(-1,1)$ and let $f \in S Q(\alpha)$. Then $f$ is convex for $|z|<1 / 3$, if $\alpha=0$, and

$$
|z|<\frac{-C+\sqrt{C^{2}+36|\alpha|}}{6|\alpha|}
$$

where $C=2(3-\alpha)+|\alpha|+3$, if $\alpha \neq 0$.

Proof. Let $f \in S Q(\alpha)$. Then

$$
\sqrt{f^{\prime}(z)} \prec \frac{3}{3+(\alpha-3) z-\alpha z^{2}}
$$

and there exists a Schwarz function $w(z)$ with $w(0)=0$ and $|w(z)|<1$ such that

$$
\sqrt{f^{\prime}(z)}=\frac{3}{3+(\alpha-3) w(z)-\alpha w^{2}(z)} .
$$

Then

$$
1+\frac{z f^{\prime \prime}(z)}{f^{\prime}(z)}=1+\frac{-2(\alpha-3) z w^{\prime}(z)+4 \alpha z w(z) w^{\prime}(z)}{3+(\alpha-3) w(z)-\alpha w^{2}(z)} .
$$

Hence

$$
\left|\frac{z f^{\prime \prime}(z)}{f^{\prime}(z)}\right|=\left|z w^{\prime}(z)\right| \frac{|2(3-\alpha)+4 \alpha w(z)|}{|1-w(z)||3+\alpha w(z)|}
$$

Using the well-known estimate [7]:

$$
\left|w^{\prime}(z)\right| \leq \frac{1-|w(z)|^{2}}{1-|z|^{2}},
$$

We have from (9)

$$
\left|\frac{z f^{\prime \prime}(z)}{f^{\prime}(z)}\right| \leq \frac{|z|(1+|w(z)|)(2(3-\alpha)+4|\alpha||w(z)|)}{\left(1-|z|^{2}\right)(3-|\alpha||w(z)|)}
$$

Hence if

$$
\frac{|z|(1+|w(z)|)(2(3-\alpha)+4|\alpha||w(z)|)}{\left(1-|z|^{2}\right)(3-|\alpha||w(z)|)}<1,
$$

Then $f$ is convex. So it is enough to find the condition of $|z|$ to satisfy the inequality (10). In case $\alpha=0$, then inequality (10) reduces to

$$
\frac{2|z|(1+|w(z)|)}{1-|z|^{2}}<1
$$

And (11) is satisfied for $|z|<1 / 3$, since $|w(z)|<|z|$. Hence we can conclude that $f$ is convex for $|z|<1 / 3$, in case $\alpha=0$. Now we suppose that $\alpha \neq 0$ and let $|w(z)|=R$ and $|z|=r$. And let us put

$$
\begin{aligned}
T(R)= & 4|\alpha| r R^{2}+\left(2(3-\alpha) r+4|\alpha| r+|\alpha|-|\alpha| r^{2}\right) R \\
& +2(3-\alpha) r-3+3 r^{2}
\end{aligned}
$$

Now

$$
T^{\prime}(R)=8|\alpha| r R+2(3-\alpha) r+4|\alpha| r+|\alpha|-|\alpha| r^{2}=0
$$

implies

$$
R=R_{1}=-\frac{2(3-\alpha) r+4|\alpha| r+|\alpha|\left(1-r^{2}\right)}{8|\alpha| r}<0 .
$$

And $T(0)<0$ is equivalent to

$$
r<\frac{-(3-\alpha)+\sqrt{\alpha^{2}-6 \alpha+18}}{3}:=r_{0} \text {. }
$$

That is, $f$ need not be convex for $r \geq r_{0}$. And for $r<r_{0}, T(R)=0$ is equivalent to

$$
R=R_{2}=\frac{-B+\sqrt{B^{2}-16|\alpha|\left(2(3-\alpha) r-3+3 r^{2}\right)}}{8|\alpha| r} .
$$

where

$$
B=2(3-\alpha) r+4|\alpha| r+|\alpha|-|\alpha| r^{2} .
$$

Put

$$
\begin{aligned}
P(r)= & 64|\alpha|^{2} r^{4}+16 B|\alpha| r^{2} \\
& +16|\alpha|\left(2(3-\alpha) r-3+3 r^{2}\right)
\end{aligned}
$$

Then

$$
P(0)=-48|\alpha|<0
$$

and 
$P(1)=64|\alpha|^{2}+32|\alpha|(3-\alpha)+64|\alpha|^{2}+32|\alpha|(3-\alpha)>0$.

Hence there exists a $r_{1} \in(0,1)$ such that $P\left(r_{1}\right)=0$ and for $0 \leq r \leq r_{1}, P(r)<0$. Hence for $0 \leq r \leq r_{1}$, $R_{2}>r, T(R)$ attains its maximum at $R=r$ for $0 \leq R \leq r \leq r_{1}$. Now

$$
\begin{aligned}
& T(r)<0 \\
& \Leftrightarrow(1+r)\left(3|\alpha| r^{2}+(2(3-\alpha)+|\alpha|+3) r-3\right)<0 \\
& \Leftrightarrow 3|\alpha| r^{2}+(2(3-\alpha)+|\alpha|+3) r-3<0 \\
& \Leftrightarrow r<\frac{-C+\sqrt{C^{2}+36|\alpha|}}{6|\alpha|},
\end{aligned}
$$

where $C=2(3-\alpha)+|\alpha|+3$, which proves Theorem 3.3.

If we put $\alpha=1 / 2$ in Theorem 3.3, we can get next Corollary.

Corollary 3.2. Let $f \in S Q(1 / 2)$. Then $f$ is convex for

$$
|z|<(-17+\sqrt{389}) / 6=0.453847 \cdots \text {. }
$$

\section{Acknowledgements}

The research was supported by Kyungsung University Research Grants in 2012.

\section{REFERENCES}

[1] J. Sokół, "A Certain Class of Starlike Functions," Computers and Mathematics with Applications, Vol. 62, No. 2, 2011, pp. 611-619. doi:10.1016/j.camwa.2011.05.041

[2] M. S. Rovertson, "Certain Classes of Starlike Functions," Michigan Mathematical Journal, Vol. 76, No. 1, 1954, pp. 755-758.

[3] W. Janowski, "Extremal Problems for a Family of Functions with Positive Real Part and Some Related Families," Annales Polonici Mathematici, Vol. 23, 1970, pp. 159177.

[4] W. Janowski, "Some Extremal Problems for Certain Families of Analytic Functions," Annales Polonici Mathematici, Vol. 28, 1973, pp. 297-326.

[5] R. Jurasiska and J. Stankiewics, "Coefficients in Some Classes Defined by Subordination to Multivalent Majorants," Annales Polonici Mathematici, Vol. 80, 2003, pp. 163-170.

[6] S. S. Miller and P. T. Mocanu, "Differential Subordinations, Theory and Applications," Series of Monographs and Textbooks in Pure and Applied Mathematics, Vol. 225, Marcel Dekker Inc., New York, 2000.

[7] P. Duren, "Univalent functions, A Series of Comprehensive Studies in Mathematics," Vol. 259, Springer-Verlag, New York, 1983. 